\title{
Essais
}

Revue interdisciplinaire d'Humanités

Hors-série 3 | 2016

Usages critiques de Montaigne

\section{Pour une approche sociologique de Montaigne}

\section{Philippe Desan}

\section{(2) OpenEdition}

Journals

Édition électronique

URL : http://journals.openedition.org/essais/6979

DOI : 10.4000/essais. 6979

ISSN : 2276-0970

\section{Éditeur}

École doctorale Montaigne Humanités

\section{Édition imprimée}

Date de publication : 1 novembre 2016

Pagination : 126-145

ISBN : 979-10-97024-00-0

ISSN : 2417-4211

\section{Référence électronique}

Philippe Desan, « Pour une approche sociologique de Montaigne », Essais [En ligne], Hors-série 3 | 2016, mis en ligne le 26 février 2021, consulté le 18 mars 2021. URL : http://journals.openedition.org/ essais/6979; DOl : https://doi.org/10.4000/essais.6979 


\section{Pour une approche sociologique de Montaigne}

\section{Philippe Desan}

Montaigne est souvent présenté comme un auteur qui échappe à toute catégorisation. Pour beaucoup, il symbolise l'exception par excellence : un auteur idiosyncrasique qui ne permet pas la généralisation. Sa singularité serait même la preuve de son génie. D'autres voient en lui le reflet d'une idéologie naissante - voire renaissante - qui précisément met en avant un individu pratiquement autonome vis-à-vis des structures sociales et politiques de son temps, lui reconnaissant même la capacité à comprendre le monde et la société à partir de ses propres expériences. Dans ce cas, l'accent est presque toujours mis sur l'originalité et l'indépendance de sa personne : Montaigne comme individu exceptionnel. L'historien de l'art Jacob Burkhardt a défini la Renaissance comme l'invention de l'individu, avec tout ce que cela sousentend de mise en scène et de Self-fashioning, pour reprendre l'expression de Stephen Greenblatt ${ }^{1}$. Cette conception libérale de la Renaissance où le sujet s'affranchit des dogmes et revendique son autonomie et sa liberté face aux systèmes de pensée dominants fait effectivement de Montaigne un auteur privilégié dans la mesure où l'essai, en tant que genre inventé par cet auteur, présuppose une forme de consubstantialité entre l'homme et l'œuvre.

On pourrait donc conclure que jamais une œuvre n'a existé de façon si personnelle et si indépendante de son contexte social et politique. Bref, les Essais se suffiraient à eux-mêmes et, comme le soutenait d'ailleurs le docteur Payen, éminent " montaignologue » du XIXe siècle, la biographie de l'auteur et tout ce qui concerne sa vie publique n'apporteraient absolument rien à la compréhension et à l'interprétation de l'œuvre, car l'homme est entièrement visible dans son livre ${ }^{2}$. Cette approche psychologisante de la création littéraire ignore l'influence des croyances et des pratiques sociales sur des décisions et des

1 Stephen Greenblatt, Renaissance Self-fashioning from More to Shakespeare, Chicago, University of Chicago Press, 1980.

2 Bnf, Fonds Payen, Z. 649, f. 17v. 
comportements que nous avons trop tendance à concevoir comme personnels et singuliers. De plus, une telle position assume que les concepts traversent les siècles sans aucune modification de sens, tout comme si les idées représentaient des universels non affectés par leur contexte social et historique. Dans tous les cas, ce qui est systématiquement mis en avant est l'autonomie de la pensée libre et individuelle. L'idéologie qui nous habite nous oblige à rechercher la singularité, car elle valorise l'originalité comme seul critère artistique et philosophique digne de considération.

\section{Institutions et habitudes collectives}

C'est précisément ce rapport problématique entre l'auteur et la société que nous proposons d'aborder ici à partir de l'exemple de Montaigne. Nous mettons en avant l'auteur et non pas les Essais, car notre approche vise à montrer comment Montaigne-auteur fut aussi un acteur social dont la création littéraire ou philosophique doit être comprise et étudiée au niveau sociologique, c'est-à-dire dans un cadre de contraintes collectives qui façonnent des choix particuliers. Les Essais s'inscrivent donc dans un cadre prédéterminé et prévisible que l'on peut qualifier de conformiste, même si son auteur vise souvent à se démarquer de ces contraintes sociales qui structurent néanmoins sa pensée. Le père de la sociologie, Émile Durkheim, analyse cette tension entre d'un côté les croyances et les pratiques sociales d'une époque et, de l'autre, leur individualisation sous forme d'adaptation ou de variation :

\footnotetext{
De ce que les croyances et les pratiques sociales nous pénètrent ainsi du dehors, il ne suit pas que nous les recevions passivement et sans leur faire subir de modification. En pensant les institutions collectives, en nous les assimilant, nous les individualisons, nous leur donnons plus ou moins notre marque personnelle; c'est ainsi qu'en pensant le monde sensible chacun de nous le colore à sa façon et que des sujets différents s'adaptent différemment à un même milieu physique. C'est pourquoi chacun de nous se fait, dans une certaine mesure, sa morale, sa religion, sa technique. Il n'est pas de conformisme social qui ne comporte toute une gamme de nuances individuelles. Il n'en reste pas moins que le champ des variations permises est limité. ${ }^{3}$
}

Le " champ des variations " auquel se réfere Durkheim fait évidemment penser aux travaux de Pierre Bourdieu sur le champ littéraire ${ }^{4}$. Certes, du temps de Montaigne, il serait bien entendu absurde de parler d'un champ littéraire tel que l'entend Bourdieu à l'époque de Flaubert par exemple, mais on repère déjà des habitudes et des comportements liés à la publication d'un

3 Émile Durkheim, Préface à la seconde édition, Les Règles de la méthode sociologique, [1894], Paris, Presses Universitaires de France, 1977, p. xxii-xxiii.

4 Pierre Bourdieu, "Le champ littéraire ", Actes de la recherche en sciences sociales, vol. 89, $\mathrm{n}^{\circ} 1$, 1991, p. 3-46. 
livre qui nous permettent de mieux comprendre les stratégies éditoriales élaborées par Montaigne entre 1570 et $1592^{5}$. Ces stratégies sont d'ordre sociologique et politique, car elles se situent dans un système ordonné par des pratiques qui dépassent largement les simples aspirations d'un seul auteur. En ce sens, Montaigne a besoin d'être étudié au sein des réseaux, ordres, groupes et milieux auxquels il appartient à des moments déterminés de son existence.

Il ne s'agit pas de comprendre la manière dont tel auteur se représente individuellement les institutions, mais bien " la conception qu' en a le groupe ; seule, en effet, cette conception est socialement efficace ${ }^{6}$. Il faut donc partir du social et des institutions avant de considérer la place qu'occupe un individu au sein de groupes, organismes, corps d'État, clientèles, cliques ou clans. Ces organisations sociales - certaines très structurées et d'autres beaucoup plus flexibles - peuvent être définies comme institutions dans le sens large du terme. Cette conception des institutions existe comme fait social auquel est confronté l'individu qui compte intégrer ces institutions, les réformer, voire s'en démarquer. Les institutions fabriquent et reproduisent des habitus auxquels doit se plier l'individu s'il prétend y occuper une place et y jouer un rôle. Cette vision partagée des institutions - dans le sens où ses valeurs nécessitent une acceptation commune - représente le point de départ pour analyser les comportements politiques, culturels, ou tout simplement carriéristes, élaborés au sein de ces institutions. L'ensemble des actions possibles régies par les institutions correspond à des manières d'agir et des manières d'être repérables au niveau de comportements particuliers. Il s'agira donc d'étudier les habitudes collectives dans leurs expressions individuelles, car, comme l'écrit Durkheim,

[1]'habitude collective n'existe pas seulement à l'état d'immanence dans les
actes successifs qu'elle détermine, par un privilège dont nous ne trouvons pas
d'exemple dans le règne biologique, elle s'exprime une fois pour toutes dans
une formule qui se répète de bouche en bouche, qui se transmet par l'édu-
cation, qui se fixe même par écrit. Telles sont l'origine et la nature des règles
juridiques, morales, des aphorismes et des dictons populaires, des articles de
foi où les sectes religieuses ou politiques condensent leurs croyances, des codes
de goût que dressent les écoles littéraires, etc.

Les adaptations effectuées par les individus par rapport aux " normes institutionnelles " permettent certes de se démarquer de cette habitude collective, mais elles forment néanmoins le point de départ de toute action singulière et méritent pour cette raison d'être étudiées comme discours normatif contre lequel se construit et s'exprime un discours particulier. Ainsi, la rédaction et la publication des Essais - nous parlons ici du livre en tant qu'objet qui circule

5 Nous avons étudié ces stratégies éditoriales dans notre livre, Montaigne. Une biographie politique, Paris, Odile Jacob, 2014.

6 Émile Durkheim, Préface à la seconde édition, Les Règles de la méthode sociologique, p. xv.

7 Émile Durkheim, Les Règles de la méthode sociologique, op. cit., p. 9. 
sur le marché plutôt que du texte - n'échappent pas à ce fait social qui existe comme pré-texte à l'entreprise montaignienne. Rappelons que les Essais furent rédigés sur presque vingt années et sont littéralement traversés par les guerres civiles qui sévissent alors en France. Quand Montaigne publie ses Essais en 1580 ou en 1588, même ses silences sur les événements de son temps interpellent ses lecteurs qui consomment un texte en fonction d'attentes et d'engagements historiquement déterminés. C'est en ce sens qu'un texte échappe toujours à son auteur pour rejoindre le cadre plus général des institutions dans lequel il se présente.

Écrire, traduire ou publier un livre répond à des attentes et engendre des projections fortement influencées par le cadre social et culturel lié au monde du livre et à son organisation. Publier un livre durant la seconde moitié du $\mathrm{XVI}^{\mathrm{e}}$ siècle répond par exemple à des pratiques codifiées auxquelles doit se conformer un auteur. C'est en étudiant ce " conformisme " social auquel est confronté Montaigne que nous pourrons ensuite définir la nature particulière des Essais qui doivent être abordés dans leur rapport à la norme définie par les pratiques et les attentes sociales d'une époque. La forme même de l'essai peut ainsi être comprise comme un genre qui, bien que créé par Montaigne, représente néanmoins une façon de voir et de parler d'un monde en crise. Ainsi, l'approche qui consiste à faire de Montaigne un écrivain isolé dans sa tour, s'interrogeant sur la condition humaine tout en regardant jouer sa chatte, relève de l'image d'Épinal. Cette singularisation d'un auteur retiré du monde nuit considérablement à la compréhension du texte des Essais, car ce soi-disant isolement du sujet vis-à-vis des institutions de son temps paraît en effet des plus problématiques quand on considère la biographie de Montaigne. C'est pour cette raison qu'il semble important de replacer la production et la lecture des Essais dans le cadre des institutions (parlement de Guyenne, mairie de Bordeaux, corps diplomatique, etc.) qui précèdent et contraignent forcément la production écrite d'un auteur. Les manifestions qui se présentent comme les plus personnelles et les plus privées sont elles-mêmes influencées et régies par la norme du modèle collectif. Une fois de plus, Durkheim s'était interrogé sur le rapport entre l'individu et le social :

Quant à leurs manifestations privées, elles ont bien quelque chose de social, puisqu'elles reproduisent en partie un modèle collectif; mais chacune d'elles dépend aussi, et pour une large part, de la constitution organico-psychique de l'individu, des circonstances particulières dans lesquelles il est placé. ${ }^{8}$

C'est à ce point qu'intervient la biographie de l'auteur, non pas comme moyen permettant d'expliquer l'acte littéraire ou la pensée philosophique, mais bien comme élément constitutif et structurant des institutions qui fait agir ou réagir l'individu et l'oblige à suivre des règles qui s'imposent à lui comme 
habitus. Comme le souligne Durkheim, c'est en effet un état du groupe qui se répète et se perpétue chez l'individu. Ces pratiques possibles nous sont transmises par les générations qui nous précèdent et s'imposent à nous comme des règles collectives. Ces façons de faire représentent des phénomènes sociaux qui peuvent être étudiés de façon objective. Elles forment la base de la structure politique d'une société, car elles sont « la manière dont différents segments qui la composent ont pris l'habitude de vivre les uns avec les autres" ". Chaque pratique sociale liée à un groupe, un organisme, un ordre ou une classe peut ainsi être considérée comme une institution à part entière. Et Durkheim de conclure ainsi le premier chapitre des Règles de la méthode sociologique où il propose une définition des faits sociaux : "Est fait social toute manière de faire, fixée ou non, susceptible d'exercer sur l'individu une contrainte extérieure; ou bien encore, qui est générale dans l'étendue d'une société donnée tout en ayant une existence propre, indépendante de ses manifestations individuelles ${ }^{10} "$.

Comme nous l'avons proposé, c'est donc bien dans le sens large du terme qu'il faut ici comprendre les institutions. Selon le sociologue, on peut en effet, sans dénaturer le sens de cette expression, appeler institution, " toutes les croyances et tous les modes de conduite institués par la collectivité ; la sociologie peut alors être définie : la science des institutions, de leur genèse et de leur fonctionnement ${ }^{11}$ ». Il faut pourtant reconnaitre que les institutions analysées par Durkheim, c'est-à-dire celles de la troisième République, ne correspondent évidemment pas à celles du XVI e siècle. Pourtant, il faut reconnaître que l'Ancien Régime possède des institutions souvent plus structurées, précisément parce qu'elles dépendent largement des trois ordres qui régissent tout comportement social et politique. C'est donc de façon sui generis (une approche chère à Durkheim) que nous proposons d'adapter ce concept d'institution à la société du $\mathrm{XVI}^{\mathrm{e}}$ siècle. Ainsi, la démarche recommandée par Durkheim reste à notre avis valide.

Revenons sur l'exemple de la publication d'un livre à la Renaissance. C'est une opération qui suit des règles institutionnalisées (comme l'obtention d'un privilège par exemple), mais répond également à des pratiques et des attentes elles-mêmes déterminées par des groupes de pression souvent en compétition les uns avec les autres. Les travaux de Roger Chartier ont sur ce point mis en lumière des comportements et des pratiques essentiellement sociologiques ${ }^{12}$. Ainsi, dans le cas qui nous intéresse ici, par institution il faut non seulement comprendre le processus de publication d'un livre à la Renaissance, c'est-à-

$9 \quad$ Ibid., p. 13.

10 Ibid., p. 14. Le texte est en italiques chez Durkheim.

11 Émile Durkheim, préface à la seconde édition, in Les Règles de la méthode sociologique, p. xxii.

12 Voir notamment, La main de l'auteur et l'esprit de l'imprimeur: XVI'-XVIII siècle, Paris, Gallimard, coll. "Folio histoire ", 2015 ; Culture écrite et société : l'ordre des livres, XIVEXVII siècle, Paris, Albin Michel, 1996. 
dire le circuit de production et de diffusion d'un objet conçu pour un public, quel que soit sa taille, mais également l'intention de l'auteur et l'effet recherché dans le cadre des pratiques sociales et culturelles de la fin de la Renaissance. Publier un livre n'est en effet pas un acte anodin. Généralement, l'impression d'un ouvrage répond non seulement à des conventions partagées par un milieu, mais permet aussi à un individu de se positionner - et ainsi d'être reconnu dans un ensemble plus large de pratiques académiques, artistiques, littéraires ou philosophiques; sans parler bien entendu du poids politique qu'un livre possède nécessairement par son acceptation ou son rejet institutionnel.

Publier un livre à la Renaissance représente donc un fait social à part entière - je renvoie sur ce point au beau livre de Natalie Zemon Davis sur le don, et plus particulièrement à sa conférence effectuée à la Royal Historical Society en 1983 sur le livre en tant que présent au XVI ${ }^{\mathrm{e}}$ siècle ${ }^{13}$. Éditer, imprimer et publier un livre exprime une forme d'action sociale, car la diffusion d'un texte implique la propagation d'idées dans des circuits et des réseaux déjà constitués. Bien plus qu’à notre époque - ceci est dû au fait que les marchés et circuits de distribution sont beaucoup plus limités au XVI siècle -, un livre s'inscrit dans une stratégie éditoriale maîtrisée par les libraires et les éditeurs. L'auteur, tout comme le libraire d'ailleurs, est presque toujours conscient d'une réception qu'il tente de maîtriser en anticipant les réactions d'un public lui-même influencé par ce que nous appellerons le « hors-texte ». C'est là toute l'importance du paratexte (préface, prologue, avis au lecteur, avis de l'éditeur, index, vie de l'auteur, etc.) à la Renaissance ${ }^{14}$. Publier un ouvrage implique une manière d'agir profondément sociale. Elle permet de se faire une place au sein d'un groupe (on pense ici aux stratégies d'inclusion et d'exclusion au sein du groupe des poètes de la Pléiade par Ronsard), ou encore de progresser dans la hiérarchie d'une institution (ouvrages de droit ou de médecine par exemple), ou tout simplement de se faire mieux connaitre pour jouir d'une plus grande visibilité avant de prétendre faire carrière dans un des corps constitués (c'est le cas de Montaigne en 1580) ${ }^{15}$. Faire imprimer un objet-livre (même à compte d'auteur, comme c'est encore le cas de Montaigne en 1580) ne doit pour ces raisons pas être considéré comme un acte strictement singulier (et sur ce point - de façon tout à fait symptomatique - Montaigne se défend de ne vouloir écrire que pour ses parents et amis), mais aussi comme un fait social.

13 Cette étude a été reprise dans Essai sur le don dans la France du XVIe siècle, Paris, Seuil, 2003.

14 Sur l'importance du paratexte des Essais, voir notre article : "Paratexte des Essais ", in Dictionnaire de Michel de Montaigne, éd. Philippe Desan, Paris, H. Champion, 2007, p. 861-863.

15 Dans notre biographie politique de Montaigne, nous avons proposé l'idée que la première édition des Essais de 1580 doit aussi être considérée comme un curriculum vitae permettant à son auteur de mettre en avant sa connaissance du monde diplomatique auquel il aspire. 
Dans Les Règles de la méthode sociologique, Durkheim précise ce qu'il entend par "fait social ": "ils [les faits sociaux] consistent en des manières d'agir, de penser et de sentir, extérieures à l'individu, et qui sont douées d'un pouvoir de coercition en vertu duquel ils s'imposent à lui ${ }^{16}$ ». Ces types de conduites sont extérieures à l'individu et se présentent sous forme de normes ; elles le contraignent à penser et à agir dans un cadre prédéfini. Cette force tranquille des faits sociaux pousse naturellement l'auteur vers une forme de conformisme institutionnel. Ce conformisme s'impose inconsciemment à l'individu qui agit certes singulièrement, mais toujours dans un rapport de forces qu'il ne maitrise pas et dont il dépend nécessairement. Cette force coercitive imposée au niveau des comportements sociaux possibles doit être étudiée dans son rapport aux actions individuelles : elle les détermine, les influence et les conditionne. On voit dès lors que des choix qui, au premier abord, pourraient paraitre singuliers sont en fait définis par des pratiques sociales élaborées et acceptées par un groupe, un ordre, une classe ou une clientèle. Ce modèle collectif est toujours présent et doit servir de point de référence à toute action personnelle. Il permet de situer Montaigne dans des réseaux et des circuits plus larges qui certes intègrent ses aspirations personnelles, mais l'autorisent également à adopter des positions parfois en contradiction avec l'habitus lié à un groupe ou un ordre.

Sans être nécessairement conscient que ses actions relèvent d'habitus (ou d'habitudes collectives selon Durkheim) - ce qui n'exclut pas qu'il puisse s'identifier à un groupe, à une clientèle ou même opter pour des alliances passagères parfois contre nature -, Montaigne s'exprime cependant au nom d'une entité constituée, à partir d'un discours codifié par le public ou le lectorat auquel il s'adresse et dans lequel il évolue. Ces pratiques constituées définissent nos choix individuels et délimitent les possibilités d'action de chacun. À partir de cette constatation, il devient possible d'aborder les actions singulières (liée à l'écriture, la publication et la diffusion d'un livre) à partir d'une approche sociologique. Sous le vernis des institutions, et parfois en contradiction avec les ordres, les groupes ou les clientèles auxquels ils appartiennent, les individus possèdent aussi des aspirations personnelles qu'il ne faut pas négliger ou sous-estimer pour autant.

\section{Idéologie, adaptation et justification}

Le concept d'idéologie - non pas dans le sens originellement forgé par Cabanis et Destutt de Tracy, puis modifié par Marx, mais plutôt à partir de l'acception proposée par Louis Althusser - est aussi utile à l'approche sociologique de Montaigne, car «l'idéologie représente le rapport imaginaire

16 Émile Durkheim, Les Règles de la méthode sociologique, op. cit., p. 5. 
des individus à leurs conditions réelles d'existence ${ }^{17}$ ». On sait sur ce point que Montaigne projette souvent ses imaginations sur la réalité au point de confondre les deux. L'idéologie sous-entend donc une projection imaginée par rapport à une réalité déformée par des aspirations individuelles. Althusser explique que "ce n'est pas leurs conditions d'existence réelles, leur monde réel, que les 'hommes' 'se représentent' dans l'idéologie, mais c'est avant tout leur rapport à ces conditions d'existence qui leur y est représenté ${ }^{18}$ ". Nous sommes donc bien dans le domaine d'une représentation imaginée de réalité des institutions dont nous parlions plus haut. Cette représentation déformée s'impose alors comme un idéal vers lequel tend l'individu. Nous quittons pour un temps la conception strictement objective des faits sociaux définis par Durkheim pour prendre en compte des faits moins tangibles qui ont à voir avec l'ambition, les aspirations, voire les fantasmes issus d'une représentation imaginée et idéalisée des institutions comme faits sociaux. Cette distinction a pour avantage de réintroduire la subjectivité dans un modèle sociologique en accordant une importance à l'imaginaire conçu comme réalité par l'individu. Peu importe si cette réalité imaginée résulte d'une fausse conscience. Au contraire dirons-nous, car la fausse conscience équivaut précisément à l'idéologie.

Lillusion d'un monde imaginé permet à l'individu d'agir comme si la projection de ses fantasmes et de ses désirs était réalité. Les effets de représentation et d'apparence ont ainsi autant d'importance que la réalité elle-même. C'est précisément cette fausse conscience de la réalité du monde dans lequel nous vivons et des institutions qui nous conditionnent qui nous permet d'agir contre ces mêmes institutions et de réaffirmer ainsi notre subjectivité. L'idéologie devient ainsi une force positive dans la mesure où elle permet de récupérer des projections singulières au sein d'institutions déformées par nos propres ambitions et aspirations. Althusser va dans ce sens quand il précise que " ce n'est leurs conditions d'existence réelles, leur monde réel, que les 'hommes' 'se représentent' dans l'idéologie, mais c'est avant tout leur rapport à ces conditions d'existence qui leur est représenté ${ }^{19}$ ". C'est bel et bien ce rapport entre le monde imaginé et le monde réel qui relève de l'idéologie.

Les actions humaines résultent d'une constante négociation entre, d'un côté, la normativité des institutions et, de l'autre, notre capacité à dévier de la norme pour bénéficier d'un avancement ou d'une reconnaissance au sein de ces mêmes institutions. La norme doit donc être respectée dans son

17 Louis Althusser, «Idéologie et appareils idéologiques d'État. (Notes pour une recherche) », La Pensée, nº 151, juin 1970, p. 3-38, ici p. 24. Article reproduit dans Positions, Paris, Éditions sociales, 1976.

18 Ibid., p. 25

19 Ibid. 
ensemble mais subvertie dans les actions individuelles. Montaigne agit ainsi souvent dans le cadre des institutions qu'il a rejointes (parlement, administration de la ville, corps diplomatique), adaptant son discours aux manières de faire de ces institutions. Althusser analyse ainsi les comportements humains comme faisant partie intégrante des appareils idéologiques dont ils dépendent, mais également comme libres choix (en toute conscience) effectués par le sujet qui réaffirme par la même occasion son indépendance et son autonomie vis-à-vis des institutions dont il peut même se démarquer :

L'individu en question se conduit de telle ou telle manière, adopte tel ou tel comportement pratique, et, qui plus est, participe à certaines pratiques réglées, qui sont celles de l'appareil idéologique dont 'dépendent' les idées qu'il a librement choisies en toute conscience, en tant que sujet. S'il croit à Dieu, il va à l'Eglise pour assister à la Messe, s'agenouille, prie, se confesse, fait pénitence (jadis elle était matérielle au sens courant du terme), et naturellement se repent, et continue, etc. S'il croit au Devoir, il aura les comportements correspondants, inscrits dans des pratiques rituelles, 'conformes aux bonnes mœurs'. S'il croit à la Justice, il se soumettra sans discuter aux règles du Droit, et pourra même protester quand elles sont violées, signer des pétitions, prendre part à une manifestation, etc. ${ }^{20}$

À la lecture de cette analyse, nous sommes frappés par le fait que ces remarques s'appliquent parfaitement à Montaigne et nous permettent de mieux comprendre la profonde contradiction inhérente aux Essais qui tantôt montrent Montaigne comme un conservateur et un conformiste et tantôt comme un individu toujours prêt à transgresser les règles et le décorum de son époque. Comment expliquer cette dualité entre le réel et l'imaginaire ? Cette tension contradictoire nous semble inhérente à la forme de l'essai. En effet, l'essai pose toujours d'entrée un lieu commun (des plus conformistes) nécessaire à la prise de parole - ce sont par exemple les titres de chapitres -, alors que l'auteur détourne presque toujours ces lieux communs pour exprimer une vision subjective et imaginaire d'une réalité à laquelle il doit se référer mais qu'il transgresse néanmoins fréquemment. Le concept d'idéologie nous paraît essentiel pour aborder le texte des Essais puisqu'il nécessite une analyse qui dissocie la réalité des institutions à l'imaginaire de leur représentation par des acteurs singuliers. Comme on le voit, les actes particuliers s'insèrent toujours dans des pratiques collectives et l'étude de l'idéologie permet justement de comprendre les déformations imaginaires qui s'opèrent au niveau des individus, car " $[\mathrm{d}]$ ans l'idéologie est donc représenté non pas le système des rapports réels qui gouvernent l'existence des individus, mais le rapport imaginaire de ces individus aux rapports réels sous lesquels ils vivent ${ }^{21} »$. 
L'apport d'Althusser par rapport à Marx est ainsi d'avoir pu démontrer que " l'individu en question se conduit de telle ou telle manière, adopte tel ou tel comportement pratique, et, qui plus est, participe à certaines pratiques réglées, qui sont celles de l'appareil idéologique dont 'dépendent' les idées qu'il a librement choisies en toute conscience, en tant que sujet ${ }^{22}$ ". Mais Althusser argue cependant que l'individu fait toujours ce qu'il fait en fonction de ce qu'il croit et finit donc souvent par faire autre chose, c'està-dire à agir en dehors de la norme. Ce comportement déviant par rapport à la norme déterminée par les institutions se retrouve fréquemment chez Montaigne et les Essais comportent de multiples exemples de ces moments de dissidence $^{23}$. On pourrait ainsi dire de Montaigne que, souvent, il a " en tête d'autres idées que celles qu'il proclame ${ }^{24} »$. En définitive, Althusser rappelle qu'il n'y a d'idéologie que par le sujet qui est littéralement interpellé par l'idéologie qui le constitue concrètement en tant qu'acteur social. Jean-Paul Sartre proposait quant à lui, à la même époque qu'Althusser, une conception plus générale du terme d'idéologie. Ainsi, dans son Plaidoyer pour les intellectuels, il compare l'idéologie à " une conception globale du monde ${ }^{25}$ » et à un désir d'agir selon ses propres idées. Ces actions particulières conçues comme personnelles s'inscrivent pourtant dans des pratiques collectives dont l'individu ne peut complètement se débarrasser. Une négociation constante entre le réel et l'imaginaire serait ainsi à l'origine de toutes les actions humaines. Une conception générale d'un monde imaginé correspond assez bien à la représentation que doit nécessairement se faire un acteur qui opère au sein d'institutions auxquelles il appartient ${ }^{26}$. Peu importe si cette conception est imaginaire (fausse conscience) ou réelle puisqu' elle est, dans les deux cas, nécessaire comme référence et permet ainsi à l'individu de se positionner dans ce monde.

Dans les nombreuses carrières poursuivies par Montaigne (parlementaire, ambassadeur, gestionnaire de la cinquième ville de France, négociateur entre Henri III et Henri de Navarre, et enfin écrivain ${ }^{27}$ ), on constate qu'il s'adapte aux mondes qu'il rejoint et sait s’insérer dans des réseaux déjà orga-

22 Ibid., p. 27.

23 Voir à ce sujet le livre de Max Gauna, The Dissident Montaigne, New York, Peter Lang, 1989.

24 Louis Althusser, "Idéologie et appareils idéologiques d'État. (Notes pour une recherche) ", art. cit., p. 27.

25 Jean-Paul Sartre, Plaidoyer pour les intellectuels, Paris, Gallimard, 1972, p. 20.

26 Pour une analyse sociologique qui met l'accent sur l'idéologie bourgeoise et marchande de la fin de la Renaissance, nous renvoyons à Eric A. Johnson, Knowledge and Society. A Social Epistemology of Montaigne’s Essais, Charlottesville, Rockwood Press, 1994 ; ainsi qu’à notre livre, Les Commerces de Montaigne. Le discours économique des Essais, Paris, A.-G. Nizet, 1992.

27 Pour une approche "sociologisante " de la carrière littéraire de Montaigne, voir l'excellent livre de George Hoffmann, La Carrière de Montaigne, [2004], trad. Pierre Gauthier, Paris, H. Champion, 2009. 
nisés ; mais il n'hésite pas non plus à affirmer son indépendance vis-à-vis de ses patrons quand ceux-ci ne répondent plus à ses attentes. On pourrait dire que Montaigne passa une bonne partie de sa vie à se démarquer de groupes qu'il avait pourtant avidement cherché à intégrer ou d'individus avec lesquels il a d'abord éprouvé quelque affinité intellectuelle ou politique. Il devint expert en justification de ses choix, de ses erreurs et de lui-même en général.

Sur ce point de la justification, les travaux de Luc Boltanski et de Laurent Thevenot nous permettent de modifier les positions de Durkheim et d'Althusser afin de concevoir une construction à deux niveaux - volonté collective et intérêt particulier - qui débouche sur un "principe supérieur commun " où les individus s'adaptent constamment aux situations nouvelles qu'ils rencontrent dans des mondes différents. L'adaptation nécessaire entre la réalité collective et l'imaginaire particulier de sa représentation permet de déboucher sur un compromis satisfaisant pour l'individu ${ }^{28}$. Cette adaptation constante par rapport à une norme toujours redéfinie en fonction d'ambitions particulières permet de mieux comprendre les "déviations » de Montaigne par rapport aux institutions de son temps. Les Essais seraient ainsi le lieu par excellence où l'impératif de la justification serait réalisé. L'« Apologie de Raimond Sebond " est à notre avis l'exemple le plus flagrant de ce principe de justification chez Montaigne ${ }^{29}$. La justification d'un compromis (à la fois acceptation des règles dans leur sens général et rejet de leurs applications particulières) conduit inévitablement à une relativisation du bien commun grâce à un travail d'extériorité vis-à-vis de ce même bien commun. Dans les Essais, Montaigne pratique constamment ce mode d'interaction sociale qui lui permet de s'affranchir des autorités et des dogmes qui limitent ses possibilités d'actions sociétales et politiques.

Dans le cas de Montaigne, il semble que toute réflexion philosophique ou littéraire extraite des Essais ne peut être dissociée d'un rapport à la collectivité : la manière d'être et de penser de Montaigne exprime également une vie et une pensée collective. C'est en quoi il est difficile de dissocier philosophie, littérature et sociologie. Écoutons encore une fois Durkheim dans Les Règles de la méthode sociologique: "Est fait social toute manière de faire fixe ou non, susceptible d'exercer sur l'individu une contrainte extérieure ; ou bien encore, qui est générale dans l'étendue d'une société donnée tout en ayant une existence propre, indépendante de ses manifestations

28 Luc Boltanski et Laurent Thevenot, De la justification, Les économies de la grandeur, Paris, Gallimard, 1991.

29 Voir notre article, "Apologie de Sebond ou justification de Montaigne? ", in "Dieu à nostre commerce et société ": Montaigne et la théologie, éd. Philippe Desan, Genève, Droz, 2008, p. $175-200$. 
individuelles ${ }^{30}$ ». Cette réflexion sur les " contraintes extérieures » exercées sur une pensée individuelle au premier abord unique et novatrice s'applique particulièrement à Montaigne. Le politique et le social sont des contraintes constantes pour Montaigne qui, après 1588, finira par concevoir l'écriture comme un mode de marginalisation et donc d'indépendance par rapport à l'emprise de la vie publique. L'isolement tardif (après 1588) dans la tour marque moins un renoncement personnel qu'une obligation résultant d'un échec politique. Montaigne écrit quand la politique va mal ! L'homme est par nature dirigé par l'action ; sa réflexion reflète souvent une épreuve et une adversité non pas personnelle, mais bien publique. C'est peut-être là la leçon des Essais.

Le positionnement littéraire et philosophique de Montaigne est essentiellement déterminé par des réactions envers des stratégies liées à des ambitions politiques et sociales personnelles, même quand il prétend s'être retiré du monde. La rédaction des Essais symbolise le lieu de cette constante négociation entre les institutions et l'idée que Montaigne se fait de ces institutions. L'idéologie véhiculée par l'écriture montaignienne nous permet d'aborder les projections imaginaires effectuées par l'auteur vis-à-vis d'ambitions et de désirs bien réels mais néanmoins sans cesse négociés avec des normes qui le conduisent à défendre des positions conformistes et conservatrices et, en même temps, présenter des idées tout à fait libérales, voire révolutionnaires pour son époque. Tout retrait sous-entend une prise de position par rapport à une réalité où l'individu pense ne plus avoir sa place. Voilà peut-être pourquoi Montaigne et son livre (dans leur consubstantialité) ne se conçoivent que dans un projet existentiel qui inclut ceux qui l'entourent. Son moi n'est qu'un rapport aux autres, dans un système d'interactions à la fois singulières et sociétales dont les Essais nous permettent de cerner l'ampleur.

\section{Clientélisme et pratiques éditoriales}

C'est à partir des principes sociologiques énoncés ci-dessus que nous proposons d'aborder les œuvres de Montaigne dans le contexte des pratiques sociales et culturelles de la fin de la Renaissance. Ces pratiques sont liées aux ordres sociaux - clercs, nobles, robins, bourgeois -, mais dépendent aussi de comportements corporatistes et clientélistes -, notamment dans les milieux parlementaire, diplomatique, administratif (jurade de la ville de Bordeaux), sans oublier la moyenne noblesse de Guyenne. Impossible de faire carrière sans s'adjoindre des alliés puissants et des protecteurs qui permettront de gravir les échelons au sein de structures sociales ou professionnelles ample-

30 Émile Durkheim, Les Règles de la méthode sociologique, op. cit., p. 14. 
ment codifiées. L'auteur Montaigne existe donc dans un rapport de dépendances et de responsabilités vis-à-vis de pairs, supérieurs ou de patrons qui lui permettent d'exprimer ses ambitions politiques et sociales.

Après l'abandon de sa première carrière de parlementaire à la fin des années 1560, Montaigne fut confronté à un problème de reconnaissance au niveau régional et national. Son avenir politique dépendait de son intégration dans un système clientéliste. Au début des années 1570, une de ses préoccupations principales fut de ne pas brouiller l'image de catholique modéré et de "politique " qu'il avait réussi à se forger lors des quinze années passées à la Cour des Aides de Périgueux et au parlement de Bordeaux. Dans ses Essais de 1580, Montaigne souligne à plusieurs reprises son rôle de loyal serviteur, déterminé à suivre inconditionnellement ses patrons : " Je ne suis propre qu'à suivre, et me laisse ayséement emporter à la foulle. Je ne me fie pas assez en mes forces pour entreprendre de commander, ny guider, ny mesme conseiller : je suis bien aise de trouver mes pas trassés par autrui ${ }^{31}$ ». Montaigne fut un bon soldat, prêt à occuper les responsabilités qui lui seraient confiées, peut-être même un jour au service de l'État ${ }^{32}$.

Son entrée en littérature représente un moment important dans cette réorientation d'une première carrière de parlementaire désormais considérée comme un échec. Contrairement à ce que l'on pourrait penser, les débuts de Montaigne en tant qu'auteur (peu après la publication de la traduction de la Théologie naturelle de Raymond Sebond en 1569) n'ont rien à voir avec les Essais, puisque c'est en tant qu'éditeur qu'il profitera de la publication des œuvres de La Boétie pour glisser un texte à lui dans l'hommage rendu à l'ami disparu. Nous faisons ici référence à l'édition par Montaigne de La Mesnagerie de Xénophon publiée à Paris en 1571 alors que Montaigne venait de vendre sa charge de parlementaire à Florimond de Raemond. La publication des ouvres de La Boétie représente en effet le point de départ d'une nouvelle carrière, non pas encore littéraire, mais bien politique dans un premier temps. Le contexte de cette publication représente un cas d'école pour l'étude du système clientéliste en vigueur à cette époque. Voyons brièvement de quelle manière Montaigne organisa et découpa le petit recueil de traductions et de poésies de La Boétie, auquel il joignit sa célèbre lettre sur la mort de La Boétie, pour mieux se faire connaître et accéder à des patrons politiques potentiels.

31 Montaigne, Essais 80, II, 17, p. 459. Nous citons ici Montaigne d'après l'édition originale des Essais publiée par Simon Millanges.

32 Nadine Kuperty-Tsur, "La notion de serviteur de l'État entre éthos et pratique à la fin du XVI ${ }^{e}$ siècle en France ", in Cité des hommes, cité de Dieu : travaux sur la littérature de la Renaissance en l'honneur de Daniel Ménager, éd. Jean Céard, Marie-Christine Gomez-Géraud, Michel Magnien et François Rouget, Genève, Droz, 2003, p. 63-74. 
Commençons par l'éditeur des œuvres de La Boétie : Federic Morel ${ }^{33}$. En 1570, Morel était au sommet de sa carrière d'éditeur et d'imprimeur. C'est ainsi qu'il devint imprimeur du roi, en remplacement de Robert II Estienne, décédé peu de temps auparavant; soit un peu moins de deux mois avant l'achevé d'imprimer de La Mesnagerie de Xénophon. La publication des œuvres de La Boétie intervient logiquement après la rupture de Montaigne avec le parlement de Bordeaux ; elle règle en quelque sorte un contentieux avec le monde de la magistrature qu'il laisse derrière lui. Le voilà tourné vers un nouveau public, exclusivement princier, ou du moins lié à la haute robe des grands commis de l'État. Montaigne comprit que La Boétie pourrait lui permettre d'accéder à un milieu social qu'il ne fréquentait pas encore. Parmi les éditeurs les plus en vue à Paris, Morel avait imprimé les ouvres du chancelier Michel de L'Hospital - dédicataire que l'on retrouve pour les œuvres de La Boétie. Morel s'était dans un premier temps spécialisé dans l'édition d'opuscules touchant à l'histoire de son temps, un créneau qui semble avoir attiré Montaigne.

Morel s'était rapproché du milieu des catholiques ultra et son engagement à leurs côtés ne fléchira jamais. En 1573, l'éditeur parisien publia une apologie de la Saint-Barthélemy dont l'auteur n'était autre que Guy du Faur de Pibrac, un ami de Montaigne. Quelques années plus tard, en 1577, Morel récidivait avec une histoire apologétique des Guises comprenant des portraits de François et de Charles de Lorraine ${ }^{34}$. On constate que cet éditeur idéologiquement marqué ne fut pas choisi par Montaigne sans arrière-pensée. Ce choix représente l'aboutissement logique d'une position politique et religieuse déjà mise en évidence au moment de la publication de l'Édit de Janvier en 1562 et de nombreux textes guisards du début des années 1560. En choisissant cet éditeur, Montaigne s'identifiait ainsi à la pensée de La Boétie à un moment où il avait tout intérêt à se faire passer pour un catholique peu enclin au compromis religieux. Montaigne ne pouvait pas ignorer qu'en 1562 Morel avait imprimé plusieurs opuscules critiquant ouvertement les édits de Tolérance ainsi qu'une version catholique - largement révisionniste - du massacre de Vassy ${ }^{35}$. Cet engagement contre la tolérance envers les huguenots est d'ailleurs conforme à la position de Montaigne dans les années

33 Sur cet éditeur, voir notre étude, "La Boétie poète et ses deux éditeurs : Federic Morel et Montaigne ", in Les Poètes français de la Renaissance et leurs "libraires", éd. Denis Bjaï et François Rouget, Genève, Droz, 2015, p. 485-505.

34 Caroli Lotharingi cardinalis et Francisci ducis Guysii, litere et arma, in funebri oratione habita Nanij à N. Bocherio, theologo et ab eodem posteà latinè plenius explicata.

35 Loys Le Roy, Avertissement sur la fausseté de plusieurs mémoires, Paris, Federic Morel, 1562 ; id., Discours sur ce qui est advenu à Vassy, Paris, Federic Morel, 1562. Dans la même veine, l'année suivante, en 1563, Morel publiait Des Differens et troubles advenans entre les hommes par la diversité des opinions en la Religion, du même Loys Le Roy, ardent défenseur du statu quo politique et religieux. 
1560, une position également partagée par La Boétie. On voit donc que le choix d'un éditeur proche des idées des ultra-catholiques pour publier les œuvres de La Boétie n'est pas anodin. Montaigne comptait essentiellement toucher un lectorat fortement ancré dans le camp catholique.

Tournons-nous maintenant vers les dédicataires des textes de La Boétie soigneusement recueillis par Montaigne dans deux opuscules publiés en même temps. Qui sont-ils ? et pourquoi ont-ils été choisis ? Des questions qui n'ont pas vraiment de rapport avec les textes eux-mêmes puisque Montaigne se réservait le droit de publier les écrits politiques de La Boétie (notamment le Discours de la servitude volontaire) dans le livre (les Essais) qu'il allait bientôt commencer. Les fragments de La Boétie (des poésies et des traductions) sont tous présentés par des lettres dédicatoires adressées à des personnages importants qui appartiennent tous au même milieu de la haute robe. La stratégie éditoriale élaborée par Montaigne, grâce à une fragmentation des écrits de La Boétie, a pour but de diffuser son propre nom et d'acquérir une visibilité qu'il ne possède pas encore à cette époque. Montaigne sélectionne pour cette occasion un petit nombre de dédicataires, tous triés sur le volet et qui partagent avec lui ce que nous pourrions appeler une même "vue idéologique » du conflit religieux qui faisait désormais rage en France.

Le premier de ces dédicataires est Louis de Lusignan de Saint-Gelais, seigneur de Lansac (1513-1589). On peut considérer Lansac comme le dédicataire le plus important du premier opuscule conçu par Montaigne dès 1570. Les titres et les qualités de ce personnage - conseiller de l'ordre du roi, conseiller en son Conseil privé, surintendant de ses finances et capitaine de cent gentilshommes de sa maison - sont dûment énumérés au début de l'épître dédicatoire. Montaigne transforme ainsi la traduction de La Boétie en un "present qui m’a semblé vous estre propre ${ }^{36}$ ". Dans son épître, Montaigne se livre à un éloge hyperbolique de l'ami disparu avant de révéler à son illustre dédicataire ses préoccupations présentes. Il avoue son désir de lui offrir quelque chose "en recognoissance des obligations que je vous doy, et de l'ancienne faveur et amitié que vous avez portee à ceux de nostre maison ». Montaigne s'inscrit ainsi sans détour dans un système de dépendance clientéliste (" obligations que je vous dois ») et suggère à Lansac qu'il peut compter sur lui pour continuer de servir son nom et sa maison, réitérant fidélité et subordination envers une famille de la haute noblesse. Montaigne rappelle sa disponibilité et offre ses services à ce personnage d'État. La correspondance de Lansac met en avant l'ampleur de ses réseaux et son influence à la cour. Il était en relation avec la plupart des princes d'Europe, plus particulièrement en Italie, et avait le pouvoir de recevoir Montaigne dans son réseau de clients.

36 "A Monsieur Monsieur de Lansac", Mesnagerie de Xénophon, in Euvres complètes d'Estienne de La Boétie, éd. Paul Bonnefon, Bordeaux/Paris, G. Gounouilhou/J. Rouam, 1892, p. 63. 
Le second dédicataire des œuvres de La Boétie fait également partie du Conseil privé du roi et compte parmi les hommes les plus en vue du royaume au début des années 1570. Henri de Mesmes (1531-1596), seigneur de Roissy et de Malassise, appartient à la génération de Montaigne. À la fin de l'année 1569, Lansac et Mesmes - avec Morvilliers, Pellevé, L'Aubespine et Birague - avaient été nommés au Conseil des affaires du royaume, un groupe très restreint de six hommes responsables des finances royales. La vie de Henri de Mesmes, marquée par une succession de retraits dans la vie privée et de retours en politique et au service du royaume, offre un parcours atypique que Montaigne pressentait peut-être pour lui-même. Montaigne s'adresse à Henri de Mesmes en toute complicité, lui rappelant qu'ils partagent la même vision d'une politique où la dissimulation doit faire place à la transparence : "De ma part j'ayme mieulx estre plus à mon aise, et moins habile ; plus content, et moins entendu ${ }^{37}$ ». Cette considération personnelle fait rapidement place à un "nous " complice qui associe explicitement les deux hommes dans une approche commune des affaires publiques : "Voylà pourquoy, Monsieur, quoy que des fines gens se mocquent du soing que nous avons de ce qui se passera icy apres nous ". Montaigne reprend à son compte une idée déjà développée par Henri de Mesmes qui, lui aussi, estimait que la postérité ne se juge pas à la renommée passagère d'un homme. Peu importent les jugements incertains de l'opinion publique, Montaigne et Henri de Mesmes se situent dans une temporalité différente, car ils possèdent une conception identique de la politique. C'est du moins l'idée émise par Montaigne dans son épître dédicatoire à Henri de Mesmes. Comme pour Lansac, la lettre de Montaigne se termine aussi par une demande de service faite dans le même esprit et avec la même intention : "Ce me sera tousjours honneur de pouvoir faire chose qui revienne à plaisir à vous ou aux vostres, pour l'obligation que j'ay de vous faire service ${ }^{38}$ ".

Le troisième dédicataire des œuvres de La Boétie est Paul de Foix (15281584), comte de Carmaing, homme d’Église et diplomate. Il était lui aussi bien en cour au début des années 1570. De cinq ans l'aîné de Montaigne, Paul de Foix occupa les fonctions d'aumônier de Catherine de Médicis et de conseiller-clerc au parlement de Paris. C'était aussi un homme politique réputé pour sa magnanimité. Montaigne décida de dédier à Paul de Foix vingt-cinq sonnets de La Boétie publiés séparément dans un second opuscule intitulé Vers François de feu Estienne de La Boetie. Ce choix aurait pu porter ses fruits, car Paul de Foix était certainement le mieux placé des trois dédicataires pour conforter Montaigne dans ses ambitions diplomatiques. Dans la pièce liminaire qui sert d'introduction aux sonnets de La Boétie, Montaigne

37 "A Monseigneur Monsieur de Mesmes ", Mesnagerie de Xénophon, p. 159.

38 Ibid., p. 160. 
loue les mérites de cette vieille famille gasconne à laquelle il s'associe par son parler vif et sans vergogne. Il rappelle à Paul de Foix le lien de la terre qui les unit " au reng de la premiere maison de Guyenne, receu de vos ancestres, avez adjousté du vostre le premier reng encore en toute façon de suffisance ${ }^{39}$ ». Montaigne propose à son tour de servir les intérêts de son patron et de sa région.

Le dernier dédicataire est Michel de L'Hospital (1507-1573) à qui Montaigne adresse la poésie latine de La Boétie. Il représente un cas à part. Montaigne avait rencontré le chancelier de France à Bordeaux lors d'un lit de justice en 1565 et il appréciait en lui le poète néo-latin humaniste qui avait occupé les plus hautes fonctions de l'État. Lorsque Montaigne lui dédie les Poemata de La Boétie, l'ancien chancelier de France venait de publier un Mémoire sur la nécessité de mettre un terme à la guerre civile (1570), sujet d'actualité où le chancelier réitérait ses rêves de tolérance et de concorde religieuses. Son échec à élaborer un concile national et la colère suscitée par la paix de Longjumeau signée le 23 mars 1568 avaient précipité son départ du gouvernement. Montaigne est conscient que le chancelier vient d'entrer en discrédit après avoir restitué les sceaux royaux au cours de l'année 1568. Il décide néanmoins d'afficher sa fidélité dans les moments difficiles. On sait que la fidélité et la loyauté seront précisément les deux qualités essentielles mises en avant par l'auteur des Essais en 1580. Ces deux qualités sont également au cœur des rapports de clientèle.

Grâce à ces dédicaces à des hommes bien en cour (le cas de L'Hospital étant un peu différent), Montaigne utilise l'espace préfaciel des pièces poétiques et des traductions de La Boétie pour se placer dans la lignée de son père et de ses aïeux qui furent tous de bons serviteurs du pouvoir politique en Guyenne et occupèrent jadis des charges municipales et parlementaires à Bordeaux. L'édition des ouvres de La Boétie représente certes une entrée en littérature pour Montaigne, mais la publication des deux opuscules a aussi pour but de mieux faire connaître le nom de Montaigne qui profite ainsi de La Boétie pour offrir ses services à des patrons potentiels. Cette pratique du découpage des écrits de La Boétie fut essentiellement motivée par des attentes professionnelles qui devaient faciliter l'entrée en politique de Montaigne.

La relation clientéliste évoquée dans les lettres adressées aux trois premiers dédicataires des œuvres de La Boétie suppose un échange réciproque de faveurs entre deux individus mis sur un pied d'égalité, dans un système d'aides asymétriques qui n'a rien de contractuel, mais dépend du bon vouloir de chaque parti. Le clientélisme présuppose une relation égali-

39 "A Monsieur Monsieur de Foix ", Vers françois, in CEuvres complètes d'Estienne de La Boétie, op. cit., p. 249. 
taire au niveau des individus, même s'ils sont issus de milieux différents - généralement le patron appartient à la haute noblesse et le client à la moyenne ou petite noblesse. Le rapport clientéliste - de nature strictement sociologique - nous permet ainsi de mieux comprendre l'importance de la publication des œuvres de La Boétie par Montaigne au début des années 1570, ceci à une époque où il vient de quitter la magistrature et désire intégrer les réseaux de la moyenne noblesse de province. Pour cela il lui faut trouver un " patron " qui lui permettrait d'intégrer un réseau politique fondé sur l'échange de faveurs ${ }^{40}$. L'usage politique de ce qui est donné sert de principe de base à l'échange et détermine l'utilité du système clientéliste ; mais l'utilité n'est jamais comptabilisée et ne relève pas d'une logique commerciale. Le désintéressement et le rejet de la logique marchande répondent assez bien à l'idée que se fait Montaigne des rapports humains et le système clientéliste fondé sur l'amitié et le don se retrouve souvent idéalisé dans la première édition des Essais en $1580^{41}$. Il n'existe sur ce point aucune économie de la faveur qui soit comparable aux règles et devoirs du noble et du chevalier ${ }^{42}$. En tant que système d'échange, le clientélisme reste informel et ne comporte aucun code ou mode de fonctionnement précis. Les relations sont cultivées dans le temps; chacune des deux parties possède des ressources particulières et en dispose selon son bon vouloir sous forme de dons. Ce rapport déformé (puisque son origine est d'ordre nobiliaire) et d'une autre époque (d'une autre idéologie pourrions-nous dire) perdure pourtant dans les milieux nobiliaires de la fin du XVI ${ }^{\mathrm{e}}$ siècle, notamment dans les provinces.

Le clientélisme est particulièrement efficace quand il existe une disproportion et une inégalité visible, et ressentie comme telle, entre les deux parties de ce que l'on appelle l'ordre politique du système clientéliste. Cet ordre est régi par la dépendance mutuelle entre des individus issus de milieux différents et qui n'évoluent pas dans les mêmes sphères du pouvoir. Le clientélisme facilite l'accès à des mondes socialement clos et ordinairement inaccessibles. Dans cet ordre redéfini, le client n'a jamais la prétention de s'élever au niveau de son patron, mais simplement de bénéficier d'une relation unique et privilégiée conçue comme une amitié. On retrouve

40 Sur l'importance des relations clientélistes à cette époque, voir Véronique Larcade, "La clientèle du duc d'Épernon dans le Sud-Ouest du royaume ", Annales du Midi, t. 108, n 213, 1996, p. 29-37; et surtout l'étude de Jean-François Médard, « Le rapport de clientèle, du phénomène social à l'analyse politique ", Revue Française de Science Politique, n² 26, 1976, p. 103-131.

41 Nous avons analysé la place de l'utilité chez Montaigne dans "Éléments d'une sociologie des Essais ", in Montaigne, éd. Thierry Gontier et Pierre Magnard, Paris, Éditions du Cerf, coll. "Cahiers d'histoire de la philosophie ", 2010, p. 45-66.

42 Sur les échanges de faveur à la fin du XVI e siècle, voir l'étude de Nicolas Le Roux, La Faveur du roi. Mignons et courtisans au temps des derniers Valois (vers 1547 - vers 1589), Seyssel, Champ Vallon, 2000. 
ici un thème cher à Montaigne. Patron et client participent à un échange généralisé sous forme de dons réciproques et de services échangés sans aucune comptabilité et de façon verbale. Le rapport clientéliste n'est jamais contractuel et échappe à la logique des marchés. Il est fondé sur le don réciproque. Ces dons peuvent être matériels ou symboliques; ils prennent aussi en compte l'information, qui est aussi considérée comme un objet d'échange au XVI ${ }^{\mathrm{e}}$ siècle. Nul ne songe à la valeur d'échange des biens, mais se contente de projeter ses propres besoins et ses désirs de réciprocité sur l'objet transmis gratuitement.

À une époque où le raisonnement marchand s'installe peu à peu dans les mentalités et où le nouvel ordre économique capitaliste bouleverse et remplace l'ordre féodal, Montaigne retrouve dans la relation clientéliste un idéal de noblesse révolu. Cet idéal n'est pas pour lui déplaire et il en retrouve même la trace parmi les Cannibales du Nouveau Monde. Le clientélisme dont il accepte les règles permet donc à Montaigne de conserver un mode de vie vaguement féodal tout en renforçant son affranchissement et son indépendance vis-à-vis des contraintes sociales de son temps. Peu répandu durant le Moyen Âge, le clientélisme fit son apparition dans une société où l'individu revendiquait une certaine autonomie et créait autour de lui un espace privé dont il réservait l'accès à un nombre restreint d'individus. Le développement du système clientéliste est donc intrinsèquement lié à l'épanouissement de l'individu à la Renaissance et surtout à son autonomie vis-à-vis des institutions traditionnelles que représentent les trois ordres de l'Ancien Régime. En fait, le clientélisme se présente à son tour comme une véritable institution et a donc besoin d'être étudié comme tel. Le don entraîne ainsi une redéfinition des termes de l'échange tout en conservant l'illusion d'une structure codée où les non-dits et les allusions comptent autant que les promesses. On peut dire que le clientélisme repose sur un code d'honneur tacite et jamais formulé entre deux individus ; c'est une forme de noblesse dont Montaigne apprit à s'accommoder et qu'il valorisa largement dans ses Essais.

Les divers éléments d'une sociologie de Montaigne que j'ai rapidement proposés me semblent indispensables et doivent être pris en considération avant toute réification du texte des Essais en objet littéraire ou philosophique. En ce sens, le sociologique est un présupposé du littéraire et du philosophique. Il ne s'agit nullement de dévaluer la forme ou le contenu des Essais, mais simplement de situer cet objet dans son cadre social et politique. Comme j'ai essayé de le montrer, publier un livre au $\mathrm{XVI}^{e}$ siècle contraint l'auteur 
à prendre en considération des pratiques dans lesquelles son individualité s’inscrit forcément. En 1937, Durkheim insistait sur la nécessité de ne pas perdre de vue l'individu quand on essaie de comprendre les faits sociaux d'une époque. C'est à ce point que la philosophie et la littérature rejoignent la sociologie : "C'est donc de l'individu qu'émanent les idées et les besoins qui ont déterminé la formation des sociétés, et, si c'est de lui que tout vient, c'est nécessairement par lui que tout doit s'expliquer. D'ailleurs, il n'y a rien dans la société que des consciences particulières ${ }^{43}$ ». Bien entendu, ces consciences particulières sont elles-mêmes formées au sein de consciences collectives et s'expriment toujours dans le cadre préexistant d'institutions structurées par des habitus souvent dépendants du politique et du culturel. Mes remarques sur la dimension sociologique de Montaigne et de ses Essais n'ont nullement l'intention de faire disparaître la conscience particulière de Montaigne, mais simplement de rappeler que celle-ci s'inscrit dans des faits sociaux et est façonnée par une idéologie que l'on ne peut ignorer.

\title{
Philippe Desan
}

University of Chicago

\begin{abstract}
Résumé
À partir des concepts durkheimiens de "faits sociaux ", " institutions » et « habitudes collectives ", nous développons une approche sociologique de Montaigne afin d'étudier comment les contraintes collectives façonnent les choix particuliers de Montaigne. De même, le concept d' "idéologie ", tel qu'il est redéfini par Althusser, nous permet de réévaluer le conformisme des Essais dans son rapport à la transgression des normes. Nous abordons enfin la question du clientélisme et des pratiques éditoriales chez Montaigne.
\end{abstract}

Mots-clés

Institutions, faits sociaux, habitus, idéologie, clientélisme, pratiques éditoriales.

\begin{abstract}
Starting from the Durkheimian concepts of "social facts", "institutions", and "collective habits", I develop a sociological approach to Montaigne in order to understand how collective constraints shaped the particular choices made by the author of the Essais. Likewise, the concept of "ideology", as it is redefined by Althusser, allows us to reevaluate the conformism of the Essais in relation to a transgression of the norms. Finally, I address the questions of clientelism and editorial practices of Montaigne.
\end{abstract}

\section{Keywords}

Montaigne, institutions, social facts, habitus, ideology, clientelism, editorial practices.

43 Émile Durkheim, Les Règles de la méthode sociologique, op. cit., p. 97. 